\title{
The abundance and thermal history of water ice in the disk surrounding HD 142527 from the DIGIT Herschel Key Program
}

\author{
M. Min $^{1,2}$, J. Bouwman ${ }^{3}$, C. Dominik ${ }^{2}$, L. B. F. M. Waters ${ }^{1,2}$, K. M. Pontoppidan ${ }^{4}$, S. Hony ${ }^{5}$, G. D. Mulders ${ }^{6}$, \\ Th. Henning ${ }^{3}$, E. F. van Dishoeck ${ }^{7}$, P. Woitke ${ }^{8}$, Neal J. Evans II ${ }^{9}$, and The DIGIT Team \\ ${ }^{1}$ SRON Netherlands Institute for Space Research, Sorbonnelaan 2, 3584 CA Utrecht, The Netherlands \\ e-mail: M.Min@sron.nl \\ 2 Astronomical Institute Anton Pannekoek, University of Amsterdam, Science Park 904, 1098 XH, Amsterdam, The Netherlands \\ 3 Max-Planck-Institute for Astronomy, Königstuhl 17, 69117 Heidelberg, Germany \\ 4 Space Telescope Science Institute, 3700 San Martin Drive, MD 21218, Baltimore, USA \\ 5 Universität Heidelberg, Zentrum für Astronomie, Institut für Theoretische Astrophysik, Albert-Ueberle-Str. 2, 69120 Heidelberg, \\ Germany \\ ${ }^{6}$ Lunar and Planetary Laboratory, The University of Arizona, Tucson, AZ 85721, USA \\ 7 Leiden Observatory, PO Box 9513, 2300 RA Leiden, The Netherlands \\ 8 SUPA, School of Physics \& Astronomy, University of St. Andrews, North Haugh, St. Andrews KY16 9SS, UK \\ 9 Department of Astronomy, The University of Texas at Austin, 2515 Speedway, Stop C1400, Austin, TX 78712-1205, USA
}

Received 28 November 2014 / Acceped 6 June 2016

\begin{abstract}
Context. The presence or absence of ice in protoplanetary disks is of great importance to the formation of planets. By enhancing solid surface density and increasing sticking efficiency, ice catalyzes the rapid formation of planetesimals and decreases the timescale of giant planet core accretion.

Aims. In this paper, we analyze the composition of the outer disk around the Herbig star HD 142527. We focus on the composition of water ice, but also analyze the abundances of previously proposed minerals.

Methods. We present new Herschel far-infrared spectra and a re-reduction of archival data from the Infrared Space Observatory (ISO). We modeled the disk using full 3D radiative transfer to obtain the disk structure. Also, we used an optically thin analysis of the outer disk spectrum to obtain firm constraints on the composition of the dust component.

Results. The water ice in the disk around HD 142527 contains a large reservoir of crystalline water ice. We determine the local abundance of water ice in the outer disk (i.e., beyond $130 \mathrm{AU}$ ). The re-reduced ISO spectrum differs significantly from that previously published, but matches the new Herschel spectrum at their common wavelength range. In particular, we do not detect any significant contribution from carbonates or hydrous silicates, in contrast to earlier claims.

Conclusions. The amount of water ice detected in the outer disk requires $\sim 80 \%$ of oxygen atoms. This is comparable to the water ice abundance in the outer solar system, comets, and dense interstellar clouds. The water ice is highly crystalline while the temperatures where we detect it are too low to crystallize the water on relevant timescales. We discuss the implications of this finding.
\end{abstract}

Key words. protoplanetary disks - stars: individual: HD 142527 - stars: pre-main sequence

\section{Introduction}

Ice is an important constituent in many planet formation scenarios. Ice coated grains are expected to stick together much more easily, and the enhanced surface density of solid material in the regions where water ice can exist makes forming larger structures easier. The increase of the mass in solid material in the region where there is ice compared to where there is no ice has been estimated to range from a factor of 1.6 (see, e.g., Min et al. 2011) to a factor of 4.2 (see, e.g., Thommes \& Duncan 2006). Direct observations of the icy regions in planet-forming disks and the abundance of ice in these regions are sparse. From an analysis of the Infrared Space Observatory (ISO) Long Wavelength Spectrometer (LWS) spectra of a collection of protoplanetary disks, Chiang et al. (2001) conclude that the ice features detected from two sources (confirmed by Creech-Eakman et al. 2002) can be reproduced by assuming $50 \%$ of the oxygen atoms are locked away in water ice.
HD 142527 is classified as a Herbig Ae/Be system and consists of an F-type star surrounded by a large protoplanetary disk. The disk, which extends out to beyond $1^{\prime \prime}$ in scattered light and in the millimeter continuum (which translates to $145 \mathrm{AU}$ for the $145 \mathrm{pc}$ distance to the source), basically consists of a large ring with an inner radius around $\sim 140 \mathrm{AU}$ and a heavily depleted region inside (see Rameau et al. 2012; Canovas et al. 2013; Avenhaus et al. 2014). Although the origin of the gap is uncertain, a likely possibility is the presence of a multiplanet system.

When spatially resolved, the disk is seen to be highly asymmetric in both cool dust emission (Casassus et al. 2013) and rotational molecular lines (van der Plas et al. 2014). This is attributed to azimuthal drift of large dust grains accumulated in the ring that are triggered by azimuthal variations in gas mass. These so-called dust traps are speculated to be important for planet formation (Klahr \& Henning 1997; Birnstiel et al. 2013). Multiple recent imaging studies have brought interesting features of the outer disk to light. For example, imaging polarimetry 
has revealed the presence of spiral arms (Canovas et al. 2013; Avenhaus et al. 2014) and the presence of an inner disk with a different inclination than the outer disk (Marino et al. 2015). The spiral arms are potentially formed by resonances with planets inside the gap or by gravitational instabilities in the outer disk. They have also been detected in rotational CO lines and an analysis of their characteristics suggest that they are formed by a combination of these mechanisms (Christiaens et al. 2014). To add to the intriguing complexity of the system, Biller et al. (2012) detect a companion with a separation of $12 \mathrm{AU}$ (see also Close et al. 2014, for a confirmation of this object) and a mass around $0.13 M_{\odot}$ (Lacour et al. 2016).

The disk surrounding HD 142527 has an interesting mineralogy. The composition of the innermost few AU of the disk has a very high abundance of crystalline silicates, while further out there is a higher abundance of amorphous silicates, which is similar to those in the interstellar medium (van Boekel et al. 2004).

A mineralogical analysis of the ISO SWS and LWS spectrum, from 2 to $200 \mu \mathrm{m}$, was presented in Malfait et al. (1999). These authors detected crystalline water ice along with a broad feature around $105 \mu \mathrm{m}$, which they attributed to the hydrous silicate montmorillonite. Later, similar features were identified in the ISO spectra of other sources and attributed to calcite or other forms of hydrous silicates (Chiavassa et al. 2005). Mutschke et al. (2008) disputed the identification of montmorillonite as the carrier of the broad $105 \mu \mathrm{m}$ band because at low temperatures the montmorillonite band is much narrower than the putative feature identified by Malfait et al. (1999). A detailed analysis of the mineralogy with spectra from the Spitzer space telescope, from 5 to $35 \mu \mathrm{m}$, is presented in Juhász et al. (2010).

In this paper we report on the detection of water ice with the Herschel Space Observatory in the framework of the Dust, Ice, and Gas In Time (DIGIT) Herschel Key Program (Green et al. 2016). Crystalline water ice in protoplanetary dust was tentatively detected before with Herschel in the disk around GQ Lup (McClure et al. 2012), and also in a later paper in a somewhat larger sample of T Tauri disks (McClure et al. 2015). Here we present an unambiguous detection of crystalline water ice in the disk around HD 142527. We determine the amount of ice needed to explain the spectroscopic features and compare to the elemental oxygen abundance. In addition, we revisit the ISO spectra to check for the previously claimed features and to combine the long wavelength analysis of the ice with Herschel with the strong $43 \mu \mathrm{m}$ ice feature.

The paper is organized as follows. First we introduce the new Herschel observations we secured for this source and the recalibration of the ISO data in Sect. 2. Next we outline our modeling setup in Sect. 3. The results of fitting the new data are presented in Sect. 4, followed by a discussion of the implications in Sect. 5.

\section{Summary of the observations}

\subsection{Herschel observations}

HD 142527 was observed with the Herschel photodetector Array Camera and Spectrometer (PACS; Poglitsch et al. 2010) on March 16, 2011. These observations with the unique observation identifiers (OBSID) 1342216174 and 1342216175 were taken as part of the DIGIT Herschel key program. The PACS instrument consists of a $5 \times 5$ array of $9.4^{\prime \prime} \times 9.4^{\prime \prime}$ spatial pixels (hereafter referred to as spaxels) covering the spectral range from $\sim 55-200 \mu \mathrm{m}$ with $\lambda / \delta \lambda \sim 1000-3000$. The spatial resolution of PACS, which is diffraction limited, ranges from $\sim 9^{\prime \prime}$ at $55 \mu \mathrm{m}$ to $\sim 18^{\prime \prime}$ at $200 \mu \mathrm{m}$. All DIGIT targets are observed in the standard range-scan spectroscopy mode with a grating step size corresponding to Nyquist sampling (see Poglitsch et al. 2010, for further details on PACS observing modes).

We carried out the PACS observations with four up/down scans of the grating and one nod cycle, and we used a small chopper throw. Our data were processed through the standard processing provided by the Herschel science center up to level 0. As a next step we processed our data using the Herschel Interactive Processing Environment (HIPE; Ott 2010) with track number 13.0, build 3253, using calibration version 65 and standard interactive pipeline scripts. The infrared background emission was removed using two chop-nod positions $1.5^{\prime}$ from the source in opposite directions. Absolute flux calibration was made by normalising our spectra to the emission from the telescope mirror itself as measured by the off-source positions, and a detailed model of the telescope emission available in HIPE. For a wellcentered source, the central spaxel contains the largest portion of the source flux and thus provides the highest signal-to-noise ratio $(\mathrm{S} / \mathrm{N})$ spectra. However, small pointing error drifts of the telescope can lead to flux losses and spectral artifacts. This is especially bad when analyzing (broad) dust emission bands, as such spectral artifacts can lead to spurious results. To mitigate this, we used a twofold approach. We determined the source position in time and corrected possible flux losses from small pointing drifts using the PACS beam profiles. We then extracted the spectra using the central $3 \times 3$ spaxels to be sure to recover the total flux and to minimize any spectral artifacts introduced by pointing drifts of the telescope. For well-pointed sources, the highest $\mathrm{S} / \mathrm{N}$ is reached in the central spaxel. However, a single spaxel spectrum also suffers more strongly from pointing effects than the combined $3 \times 3$ spectrum. To guarantee the best absolute flux calibration with the highest $\mathrm{S} / \mathrm{N}$ spectra, we scaled the continuum fluxes of the central spaxel spectrum to the continuum of the $3 \times 3$ spaxel spectrum using a smoothing filter with a width of a few microns. We carried out spectral rebinning with an oversampling of a factor of two and an upsampling of a factor of one corresponding to Nyquist sampling. We carried out absolute flux calibration and corrections for intrumental artifacts that were not corrected for by the model for the emission from the telescope mirror with the mean spectral response function based on repeated observations of Ceres. Using these data, we estimate that the repeatability error on the PACS spectroscopic observations is on the order of $2 \%$. The error on the absolute flux calibration is estimated to be about 4\% (see also Müller et al. 2014, for further details on flux calibration using asteroids).

In addition to the PACS spectra, we also obtained Spectral and Photometric Imaging Receiver (SPIRE) spectra from Herschel for the longer wavelengths. These spectra were previously presented by van der Wiel et al. (2014).

\subsection{Infrared Space Observatory LWS and SWS recalibration}

We have re-reduced the available Infrared Space Observatory (ISO; Kessler et al. 1996) Short Wavelength Spectrometer (SWS; de Graauw et al. 1996) and Long Wavelength Spectrometer (LWS; Clegg et al. 1996) spectra of HD 142527 (PI: Waelkens). The reasons for re-reducing these data are, first, obtaining a consistent dataset reduced entirely with the most recent version of the reduction pipelines and, second, removing biases in the overall shape of the continuum because of the way the different orders of the spectra are combined to obtain a continuous SED.

HD 142527 was observed using the SWS spectrograph on 29 Feb. 1996 (TDT10402046) in full range grating scanning 


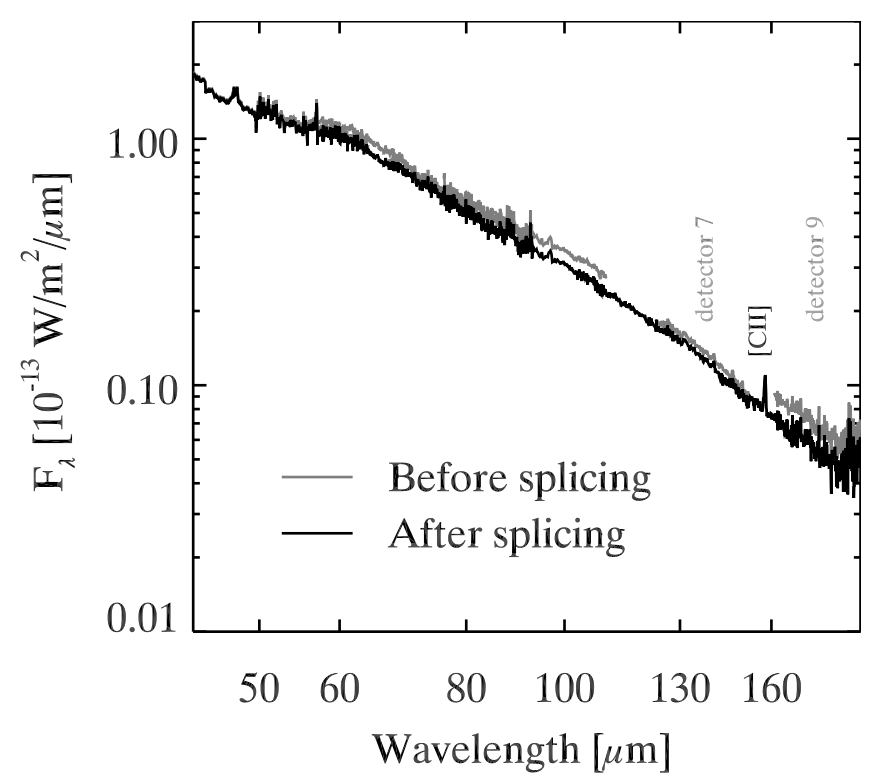

Fig. 1. HD 142527 ISO LWS spectra before and after splicing. In general the difference before and after splicing is small. We indicate the data from detectors 7 and 9 , which were shifted down by -6 and $-14 \mathrm{Jy}$, respectively, to form a continuous spectrum. The overall shape from 45 to $200 \mu \mathrm{m}$ of the dust emission is unaffected by the splicing we applied. The only line emission that is significantly detected is the [CII] at $158 \mu \mathrm{m}$.

mode (AOT01, speed 2), covering the wavelength range from 2.3 to $45 \mu \mathrm{m}$ at effective spectral resolving powers $(\lambda / \Delta \lambda)$ between 300-600. The data were reduced in the SWS Interactive Analysis environment (OSIA; de Graauw et al. 1996) using the standard pipeline (OLP version 10) up to the auto-analysis result stage, which yields separate flux and wavelength calibrated spectra for each individual detector and order. We applied extensive manual removal of bad data by clipping data that are clearly affected by glitches and baseline jumps. The cleaned spectra per detector are flat fielded, i.e., corrected for baseline differences to agree with the mean spectrum of all detectors. We applied offset corrections in cases in which the median flux of the band is less than $\sim 20 \mathrm{Jy}$, or else we applied multiplicative corrections. Finally, the cleaned and flat-fielded spectra from both observations were combined and rebinned on a common wavelength grid $(\lambda / \Delta \lambda=500)$. Prior to combining the rebinned spectral orders, we shifted band $2 \mathrm{~A}(4.1-5.3 \mu \mathrm{m})$ by $0.5 \mathrm{Jy}$ to agree with the neighboring bands.

The LWS spectrum $(45-200 \mu \mathrm{m})$ was also obtained on 29 Feb. 1996 (TDT10402250) in the full range scanning mode (LWS01). We started from the LWS auto-analysis result produced by the standard LWS pipeline (OLP version 10). Further reduction consisted of extensive bad data removal using the ISO Spectral Analysis Package and rebinning on a fixed resolution grid of $\lambda / \Delta \lambda=50$. The rebinned spectra exhibit significant offsets between data from different spectral orders, called detectors. We have corrected for these offsets in such a way that the final spliced spectra conserved as much as possible the overall slope (as measured in $\log \left(F_{\gamma}\right)$ as a function of $\log (\lambda)$ ) and shape of the broad energy distribution (see Fig. 1). No corrections have been applied to detectors 2 and 8 , detectors 0,1 and 3 required a small $(<4 \%)$ correction, while detectors 2,4 and 5 needed to be scaled down by $\sim 10 \%$. The baselines of detectors 7 and 9 are clearly discrepant. The offsets applied to these data, are -6 and $-14 \mathrm{Jy}$, respectively.

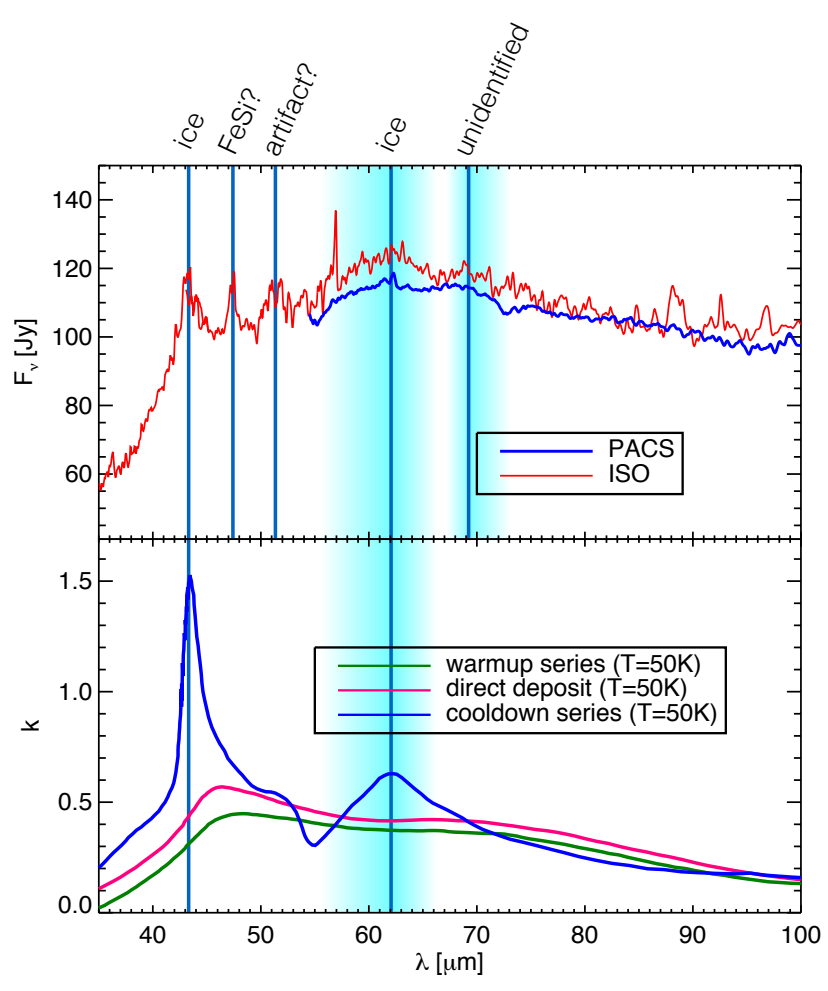

Fig. 2. Final Herschel/PACS and ISO spectra for HD 142527 (upper panel) rebinned at a spectral resolution of $R=300$ and the imaginary part of the refractive index for the different measurement series of water ice at a measurement temperature of $50 \mathrm{~K}$ (lower panel). The spectral locations of the different features visible in the spectrum are indicated. Only the cool-down series shows the crystalline ice features observed in the PACS and ISO spectra.

This approach for creating a continuous spectrum is conservative in the sense that we take care not to introduce broad features or slope changes that are not present in the data prior to combining, while splicing these independent orders. This methodology is different from that employed in an earlier reduction (Malfait et al. 1999) where it was assumed that jumps between data from different detectors in LWS were due to inaccurate removal of dark currents, the correction for which can lead to significant changes in the spectral slope. The SWS and LWS data did not need to be further scaled to form a single continuous spectrum from 2.3 to 200 micron.

The final shape of the ISO spectrum is significantly different from earlier reductions. The feature previously reported around $90 \mu \mathrm{m}$ is not detected in the recalibrated spectrum (see also Sect. 3.6). We identify calibration issues with the ISO LWS spectrum as the reason for a misidentification.

\subsection{Compositional inventory}

First we directly analyze the long wavelength spectra. We do not examine the mineralogy of the inner regions, which are probed by the Spitzer and ISO SWS/LWS spectra. For a compositional analysis of this spectral range, we refer to van Boekel et al. (2005), Juhász et al. (2010).

We plot the resulting Herschel and ISO spectra in the top panel of Fig. 2. The two features of crystalline water ice at 43 and $62 \mu \mathrm{m}$ are very prominent. In addition to these features there is a sharp feature in the ISO spectrum at $47 \mu \mathrm{m}$. A suggestion for the origin of this feature from the literature is FeSi (Ferrarotti et al. 2000), but a firm identification, including other features (for 
example, the expected FeSi feature around $32 \mu \mathrm{m}$ is not detected in the Spitzer spectrum), is currently lacking. In addition, the condensation calculations performed by Ferrarotti et al. (2000) that predict the presence of FeSi have been performed for evolved S-stars and it is in principle not expected for the elemental composition in a protoplanetary disk. There is structure in the ISO spectrum at around $51 \mu \mathrm{m}$, but it is unclear if this is simply a noisy part of the spectrum or if there is really a solid state feature. Finally, a broad feature can be seen in both the ISO and PACS spectrum around $69 \mu \mathrm{m}$. We have not been able to find an identification for this feature, but since it is visible in both the ISO and PACS spectra, we conclude it is real. Forsterite has a feature at this wavelength, but this feature is much narrower and cannot explain the broad feature detected even when we would consider a wide distribution in iron content and temperatures. The crystalline silicate diopside, $\mathrm{CaMgSi}_{2} \mathrm{O}_{6}$, has a feature with the correct width around this wavelength position, but peaking at $65 \mu \mathrm{m}$ (Chihara et al. 2001). It is therefore tempting to speculate that it is caused by a mineral with composition close to diopside, but perhaps with Fe instead of $\mathrm{Mg}$ causing the feature to shift toward the red (similar to what happens for crystalline olivine, Fabian et al. 2001).

In Sect. 3.6 we perform a more detailed compositional analysis, including the previously proposed materials on the basis of the ISO spectrum.

\section{Modeling setup}

Our main aim in this study is to constrain the composition and location of the water-ice that gives rise to the observed bands in the Herschel/PACS spectrum. These ice bands arise from the cooler, outer parts of the disk. To derive reliable parameters of the ice, however, one needs a full, realistic model of the structure of the disk that includes the ice-free inner parts. Our model partly builds on previous detailed modeling of the HD 142527 system (Verhoeff et al. 2011). Recently, the inner and the outer disk of HD 142527 were identified as not aligned (Marino et al. 2015). This causes the shadow of the inner disk on the outer disk wall to be significantly smaller than in previous models, where the inner disk is aligned. This also reflects itself in the temperature distribution of the inner edge of the outer disk. Therefore, we cannot ignore this effect in our modeling of the outer disk and we adopt a full 3D model including an inclined inner disk.

We adopt a two-step modeling approach. First, we improve on the full radiative transfer model setup by Verhoeff et al. (2011) using a 3D model setup in combination with an automatic genetic fitting algorithm to optimize the model parameters and taking the updated spectral energy distribution (SED) and new Herschel data into account. In the second step, we take the resulting size distribution of the dust grains to make a leastsquares fit to a model of optically thin emission from the spectral region with the ice features. In this second step, we assume that the thermal emission features are created in an optically thin warm surface layer and make a linear least-squares fit to a part of their spectrum assuming a single emission temperature for the feature contributing materials and a continuum made of blackbody emission. The advantage of this two-step approach is that we have a relatively robust estimate of the size distribution of the grains from the multiwavelength radiative transfer model and a detailed analysis of the mineralogy from the optically thin leastsquares analysis (see Sect. 3.6 for details).

\subsection{Multiwavelength radiative transfer modeling}

As our starting point we take the model from Verhoeff et al. (2011) with significant modifications. The basis of this model is a passive irradiated disk. We include an inner disk from the dust evaporation radius up to $13 \mathrm{AU}$, and an outer disk starting around $130 \mathrm{AU}$. The vertical structure of the gas disk is parametrized using a scale height that varies as a power law of the distance to the star. We adopt a tunable, size-dependent settling prescription based on self-consistent considerations. Each grain size is settled to its own scale height according to the settling prescription as described in Woitke et al. (2016), which is basically the prescription by Dubrulle et al. (1995) with a simple adjustment for the parameterized vertical gas structure. In the description the sedimentation is related to a single free parameter, the turbulent $\alpha$.

We construct a full radiative transfer model of the disk to compute the temperature structure and determine whether water ice is present at a given location. For this we use the temperaturedependent opacity option in the $3 \mathrm{D}$ version of the continuum radiative transfer program MCMax (Min et al. 2009). This allows us to include the temperature-dependent ice optical properties as measured by Smith et al. (1994, see also next section). The temperature-dependent opacity implementation was successfully used in, for example, Mulders et al. (2011).

The radial density distribution of the dust disk was parameterized using a radial surface density (Hughes et al. 2008)

$\Sigma(r) \propto R^{-q} \exp \left\{-\left(\frac{R}{R_{\text {exp }}}\right)^{2-q}\right\}$

for $R_{\text {in }}<R<R_{\text {out }}$. Here $R_{\text {exp }}$ is the turnover point beyond which an exponential decay of the surface density sets in and $q$ sets the power law in the inner region. Although we fix the outer radius of the disk at $2000 \mathrm{AU}$, the surface density of the disk is already highly diminished after the turnover radius, which is a fitting parameter with a typical value around a (few) hundred AU.

We use a two-component model consisting of an inner disk and outer disk. The inner disk defines the SED up to a wavelength of $\sim 15 \mu \mathrm{m}$. This part of the SED was very difficult to understand using only an inner disk in hydrostatic equilibrium, especially because of the large near-infrared excess (see Verhoeff et al. 2011, for extensive discussion on these components to the modeling), therefore a halo component was invoked that may represent a vertically extended disk wind (e.g., Bans \& Königl 2012). Here, we used a parameterized structure of the inner disk and thus find a solution without a halo, but an inner disk that is significantly extended beyond hydrostatic equilibrium. Obtaining the exact structure of the inner regions is not the aim of this paper, but it could be constrained by infrared interferometric observations. Here we use the inner disk model to get a decent fit to the SED and shadowing effects on the outer disk, which we use to constrain the composition of the outer disk component.

\subsection{Dust optical properties}

We use the Bruggeman effective medium theory to mix the various materials present in the dust (including the ice). In contrast, Verhoeff et al. (2011) simply add the opacities of pure grains. Using the effective medium theory provides a better account of the effects of mixed grain compositions and ice on the temperature structure of the disk. We take the same material composition as Verhoeff et al. (2011), based on the mineralogical analysis of 
Table 1. Composition of the dust mixture and references to the laboratory data used.

\begin{tabular}{|c|c|c|}
\hline Name & & Reference \\
\hline \multicolumn{3}{|c|}{ Silicate component \& mass fractions } \\
\hline Amorphous pyroxene & $73.2 \%$ & Dorschner et al. (1995) \\
\hline Crystalline forsterite & $8.6 \%$ & Servoin \& Piriou (1973) \\
\hline Crystalline enstatite & $14.8 \%$ & Jäger et al. (1998) \\
\hline Amorphous silica & $3.4 \%$ & Spitzer \& Kleinman (1960) \\
\hline \multicolumn{3}{|c|}{ Other components } \\
\hline Amorphous carbon & & Preibisch et al. (1993) \\
\hline Water ice & & Smith et al. (1994) \\
\hline Montmorillonite & & Koike \& Shibai (1990) \\
\hline Serpentine & & Koike \& Shibai (1990) \\
\hline Chlorite & & Koike \& Shibai (1990) \\
\hline Dolomite & & Posch et al. (2007) \\
\hline Calcite & & Posch et al. (2007) \\
\hline
\end{tabular}

$10 \mu \mathrm{m}$ spectra by van Boekel et al. (2005), but add in $25 \%$ vacuum to simulate porous grains. See Table 1 for references to the refractive index data used. Like Verhoeff et al. (2011), we use the distribution of hollow spheres (DHS; Min et al. 2005) to compute the resulting optical properties of the grains from the refractive indices obtained from the effective medium theory. We take the irregularity parameter $f_{\max }=0.8$ to properly account for particle irregularities. We adopt a continuous size distribution, $n(a) \propto a^{-p}$, where $a$ is the size of the dust grains. The sizes range from $a=0.05$ to $a=3000 \mu \mathrm{m}$.

\subsection{The location of water ice in the disk}

We set a limit on the temperature, density, and strength of the local UV radiation field to determine the location of the ice. For the temperature and pressure-dependent part of the ice condensation and sublimation, we follow the equations from Kama et al. (2009), Min et al. (2011). The vapor pressure, i.e., the equilibrium partial pressure at the sublimation temperature, is given by (after Eq. (1) in Kama et al. 2009),

$p_{v}(T)=\frac{k_{B}}{\mu m_{u}} \cdot 10^{(B-A / T)} \cdot(1 \mathrm{~K})$,

where $T$ is the temperature and $A$ and $B$ are the constants known from laboratory measurements $(A=2827.7 \mathrm{~K}, B=7.7205$; Pollack et al. 1994; Kama et al. 2009). If we assume that recondensation is proportional to the number of collisions on the surface of the grain with proportionality constant $\alpha_{\text {stick}}$, the mass increase per unit surface area per unit time of a grain becomes

$J^{+}(T)=\alpha_{\text {stick }} p \sqrt{\frac{\mu m_{u}}{2 \pi k_{B} T}}$,

where $p$ is the partial pressure of the condensing material, and $\mu m_{u}$ is the mass of a single molecule. At equilibrium $\left(p=p_{v}\right)$, i.e., when the mass loss due to thermal evaporation equals the mass increase due to recondensation, the mass loss rate per unit surface area is given by $J^{-}(T)=J^{+}(T)$.

Equation (2) was derived without the UV destruction of the ice. Assuming the ice mass loss rate due to UV photodesorption is proportional to the strength of the UV field, we obtain for the mass loss rate per unit surface area,

$J^{-}(T)=\alpha_{\text {stick }} p_{v}(T) \sqrt{\frac{\mu m_{u}}{2 \pi k_{B} T}}+\gamma^{\prime} G_{\mathrm{UV}}$ where $G_{\mathrm{UV}}$ is the local UV field strength in terms of Habing fields $\left(u_{\mathrm{UV}}^{\mathrm{Hab}}=5.33 \times 10^{-14} \mathrm{erg} \mathrm{cm}^{-3}\right.$; Habing 1968), and $\gamma^{\prime}$ is a scaling parameter. Now if we set $J^{+}(T)=J^{-}(T)$, the required minimum water vapor density for the ice to exist becomes

$$
\begin{aligned}
& \rho\left(\mathrm{H}_{2} \mathrm{O}\right)=10^{(B-A / T)} \frac{1 \mathrm{~K}}{T} \mathrm{~g} / \mathrm{cm}^{3}+\gamma G_{\mathrm{UV}} \sqrt{\frac{1 \mathrm{~K}}{T}}, \\
& \gamma=\frac{\gamma^{\prime}}{\alpha_{\text {stick }}} \sqrt{\frac{2 \pi \mu m_{u}}{k_{B}}} .
\end{aligned}
$$

We use the photochemical disk modeling code ProDiMo (Woitke et al. 2009) to estimate empirically at which UV field strength the ice is destroyed and determine the value of $\gamma$ to use. We find a value of $\gamma=10^{-20} \mathrm{~g} \mathrm{~cm}^{-3}$ corresponds well to the detailed chemistry and photodesorption rates that is computed in ProDiMo. So, for example, this means that for a typical field strength in the outer disk of $G_{U V}=100$ and a temperature of $100 \mathrm{~K}$, we need a minimum water vapor density of $\rho\left(\mathrm{H}_{2} \mathrm{O}\right) \approx 10^{-19} \mathrm{~g} \mathrm{~cm}^{-3}$ (or $n\left(\mathrm{H}_{2} \mathrm{O}\right) \approx 3300 \mathrm{~cm}^{-3}$ ) for water ice to be stable, which is significantly higher than the densities typically found in the surface layer of the disk, especially in the outer regions. Using Eq. (5), we can compute the sublimation temperature of the ice locally in the disk, also taking into account the desorption caused by UV photons. We iterate the radiative transfer and ice sublimation to arrive at a consistent solution. This treatment does not capture all the details of water chemistry and ice formation and destruction present in detailed chemical computations.

\subsection{Optical properties of water ice}

The solid state features of water ice are influenced by its formation temperature and thermal history and its current temperature. The formation temperature and thermal history mainly determine whether the ice is amorphous or crystalline and, thus, which features are present or absent. The current temperature, i.e., the temperature at which the ice emits, determines the exact wavelength positions and shapes of these features. If the ice originally condenses in amorphous form, but is subsequently heated to temperatures above $\sim 110 \mathrm{~K}$, the ice becomes crystalline, changing its spectroscopic appearance. The ice stays crystalline even if subsequently cooled down.

Smith et al. (1994) measured the temperature-dependent optical properties of water ice formed at different temperatures. They measured three different sets of optical constants: a set where the ice was condensed at $10 \mathrm{~K}$ and warmed up to the measurement temperature (series I), a set where the condensation proceeded at the same temperature at which the optical properties were measured (series II), and a set where the ice was condensed at $150 \mathrm{~K}$ and cooled down to the measurement temperature (series III). In the series I and II the ice is amorphous for temperatures lower than the crystallization temperature of $\sim 110 \mathrm{~K}$. Only in the series III measurements, where the ice was condensed in crystalline form and cooled down, are the ice crystalline at these lower temperatures. In addition, Smith et al. (1994) found a shift in the position of the $43 \mu \mathrm{m}$ crystalline ice feature with temperature, where the feature shifts to shorter wavelengths with decreasing temperature. The refractive indices as measured by Smith et al. (1994) do not directly give the right peak position for the solid state features. The peak is also dependent on the shape, size, and structure of the grains under consideration, and we model this using the grain model described in the previous section. In our radiative transfer modeling, we take 
the temperature dependence of the ice opacity into account selfconsistently. The curves for the imaginary part of the refractive index of all three series at a measurement temperature of $50 \mathrm{~K}$ are shown in the lower panel of Fig. 2.

In our modeling procedure we pick one of the three series and use the optical properties within that series that correspond to the local disk temperature at each location in the disk. Thus, we always have three different models, one where the ice was formed at lower temperatures than currently observed, one where it was formed locally, and one where it was formed at higher temperatures.

\subsection{Fitting procedure}

We use a genetic algorithm to fit the spectral energy distribution and the interferometric observations (also presented in Verhoeff et al. 2011). The algorithm we designed for this is based on the PIKAIA algorithm (see Charbonneau 1995), but revised to efficiently fit a combination of photometric and spectroscopic observations over a wide wavelength range. The possibility was added to the fitting procedure to evaluate all models generated during the fitting process to determine accurate error estimates for all fitting parameters. The error estimation process uses bootstrapping. We generate 1000 artificial observational datasets consisting of random deviations from the original dataset using the error bars on the data. For all these datasets, we compute the $\chi^{2}$ that this dataset would have with each model. This method yields a distribution of $\chi^{2}$ values that are commonly referred to as the F distribution. We determine the standard deviation, $\sigma$, of the $\mathrm{F}$ distribution. Next, we consider all models computed in the genetic fitting procedure that have a $\chi^{2}$ within $1 \sigma$ of the best-fit model. The spread on the model parameters that we find in all these models gives us the errors on the model parameters. Finally, at the end of the genetic run, we explore parameter space locally with a few hundred models centered around the best-fit parameters. This way we get a good estimate of the model uncertainties without having to compute a full scan of parameter space. For the bootstrapping method to work, the standard deviations on the observations are crucial. If these are too small, the variations in the 1000 artificial datasets are too small resulting in unrealistically small errors on the model parameters. We therefore multiply the errors on the observations by $\sqrt{\chi^{2}}$ before computing the 1000 artificial datasets. This way, when the fit is not good (i.e., $\chi^{2}>1$ ), the errors on the derived model values automatically increase. The location of the best-fit model in the parameter space is unaffected by this rescaling of the error bars; we still find exactly the same best-fit model. The only thing that is affected is the estimate of the uncertainties of the parameters.

Defining $\chi^{2}$ for an observational dataset containing both high resolution spectra (ISO and Spitzer) and photometry is nontrivial. To compute a total $\chi^{2}$, we weigh the different observational datasets differently to prevent the fitting procedure from becoming too sensitive to small parts of the spectrum simply because there are many observations in a particular wavelength region. Determining the weights to get a good fit to the spectrum is, to a certain degree, subjective and sensitive to the problem at hand. We increase the weight of the region around the ice features because in this study we are most interested in this part of the spectrum.

\subsection{Optically thin, mineralogical/chemical analysis}

From the radiative transfer modeling we get an estimate of the abundance of ice and grain size distribution. We use this as input in a linear least-squares fitting procedure. The aim of this exercise is to put firm constraints on the presence or absence of certain mineralogical or ice components in the dust. The disk is not optically thin in the far-infrared, but we assume here that the emission features originate in the optically thin surface layer of the disk and simulate the optically thick contribution with a distribution of blackbodies.

The procedure is based on that developed by Min et al. (2007) with a few small adjustments. The procedure is as follows:

1. For each component we construct a template spectrum of thermal emission at $80 \mathrm{~K}$. The template spectrum includes the best-fit mixture (silicates, amorphous carbon and water ice) and a small abundance of the component we want to test. We pick $80 \mathrm{~K}$ as the representative temperature of the inner wall of the outer disk. There is some variation in the temperature of this inner wall, but as long as we pick a single temperature for all dust components, the exact value should not significantly influence the abundance estimates.

2. We make a linear least-squares fit of the observed spectrum with this mixture plus a contribution from pure blackbody emission from a sum of blackbodies at different temperatures. For the blackbody components, we vary the temperatures between 10 and $1500 \mathrm{~K}$; the weights are determined from linear least-squares fitting.

3. To test the significance of each dust component, the abundance in step 1 is increased until the fit in step 2 gets worse by $1 \sigma$ according to a standard $\mathrm{F}$ test. This abundance is the upper limit for this component. This is carried out for the silicate and ice components in the mixture.

Following the analysis of the previous reduction of the ISO data (Malfait et al. 1999), we test for the presence of two different types of carbonates (calcite and dolomite) and three different types of hydrous silicates (montmorillonite, serpentine and chlorite). As mentioned before, we proposed these materials as components of the disk from analysis of a previous reduction of the ISO spectrum. Further, we test for the presence of the three different types of water ice corresponding to the different series of measurements by Smith et al. (1994). We determine the abundance of each of the different ice measurements, corresponding to different thermal histories (see Sect. 3.4) to track the thermal history of the ice.

\section{Results}

\subsection{Properties of the disk}

The best-fit parameters of the HD 142527 disk, as derived using the genetic fitting algorithm, are summarized in Table 2 . First we highlight one of the main findings, the ice/silicate ratio of 1.6, which is in general agreement with previously proposed values. We dedicate Sect. 5.1 to discuss this value in detail.

A few remarkable properties of the best-fit model are apparent from Table 2. First, we stress the scale height of the inner and outer disks. At the inner edge of the inner disk, the scale height expected from hydrostatic equilibrium and a midplane temperature of $1600 \mathrm{~K}$ is only $0.013 \mathrm{AU}$, which is a factor of 1.7 below the best-fit value. This reflects the problem already indicated before with the near-IR excess. At the inner edge of the outer disk, 


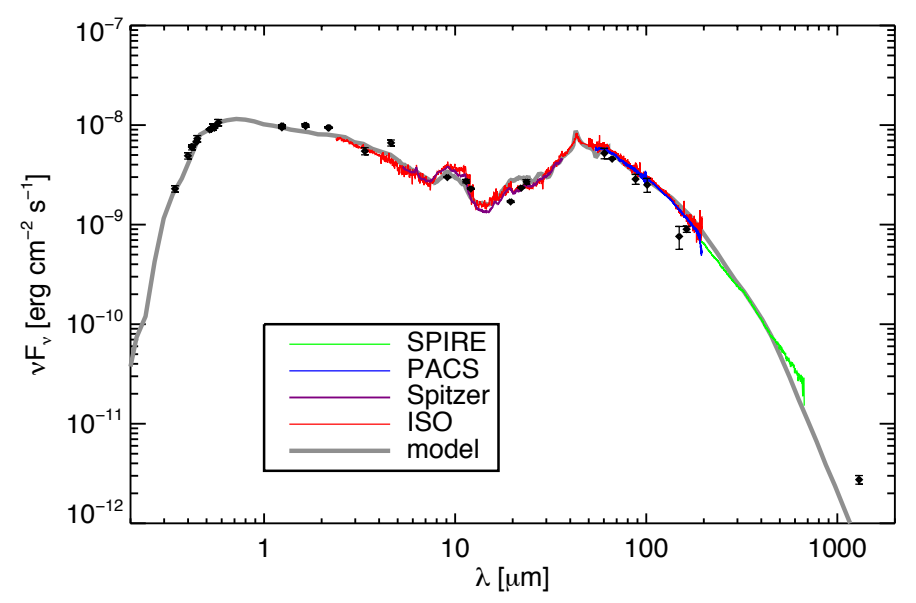

Fig. 3. Spectral energy distribution of HD 142527 together with the best-fit model.

Table 2. Parameters of the best-fit radiative transfer model.

\begin{tabular}{|c|c|c|}
\hline Parameter & Value & Error \\
\hline \multicolumn{3}{|l|}{ Inner disk } \\
\hline Inner radius: $R_{\text {in }}[\mathrm{AU}]$ & 0.38 & 0.09 \\
\hline Outer radius: $R_{\text {out }}[\mathrm{AU}]$ & 13 & fixed \\
\hline Dust mass: $M_{\text {dust }}\left[M_{\odot}\right]$ & $4.5 \times 10^{-7}$ & factor 1.6 \\
\hline Size distribution power law: $p$ & 4.9 & 0.5 \\
\hline Surface density power law: $q$ & 1.6 & 0.6 \\
\hline Scale height at the inner radius: $h[\mathrm{AU}]$ & 0.022 & 0.002 \\
\hline Scale height power law: $p_{h}$ & 1.23 & 0.08 \\
\hline Mixing strength: $\alpha$ & $1.3 \times 10^{-5}$ & factor of 2 \\
\hline Inclination: $i$ & $-46^{\circ}$ & 11 \\
\hline \multicolumn{3}{|l|}{ Outer disk } \\
\hline Inner radius: $R_{\text {in }}[\mathrm{AU}]$ & 146 & $+2 /-15$ \\
\hline Exponential radius: $R_{\exp }[\mathrm{AU}]$ & 282 & 70 \\
\hline Dust mass: $M_{\text {dust }}\left[M_{\odot}\right]$ & $1.4 \times 10^{-3}$ & factor 1.6 \\
\hline Size distribution power law: $p$ & 3.7 & 0.2 \\
\hline Surface density power law: $q$ & 1.7 & 0.5 \\
\hline Scale height at the inner radius: $h$ [AU] & 17.3 & 2 \\
\hline Scale height power law: $p_{h}$ & 1.1 & 0.1 \\
\hline Mixing strength: $\alpha$ & $4.7 \times 10^{-6}$ & factor of 2 \\
\hline Inclination: $i$ & $20^{\circ}$ & fixed \\
\hline \multicolumn{3}{|l|}{$\begin{array}{ll}\text { Global parameters } \\
\end{array}$} \\
\hline Carbon/silicate mass ratio & 0.2 & 0.1 \\
\hline Ice/silicate mass ratio & 1.6 & $+0.9 /-0.6$ \\
\hline
\end{tabular}

the scale height expected from hydrostatic equilibrium, assuming a temperature of $80 \mathrm{~K}$ is $22 \mathrm{AU}$, which is much closer to the best-fit value. This indicates that the outer disk is likely close to hydrostatic equilibrium.

Another interesting parameter is the turbulent mixing strength, $\alpha$, which we find to be much lower than the canonical value of $\alpha \sim 10^{-2}$ to $10^{-3}$ (Hartmann et al. 1998). This is surprising since the SED of this source is very red, i.e., the disk emission peaks at very long wavelengths, which classically indicates a flaring disk structure. Our model thus supports the current view that the red SED is an indication for a gapped disk structure (Maaskant et al. 2013). Finally, the total dust mass in the outer disk is relatively high. We currently assume a canonical gas-todust ratio of 100 , which implies a total disk mass of $0.14 M_{\odot}$. A possibility is that the outer disk has already lost a large portion of its gas mass. The values for the gas-to-dust ratio and turbulence parameter, $\alpha$, are degenerate in our model. To obtain the same degree of settling, but with a lower gas-to-dust ratio, we would need a higher value of $\alpha$ to keep the same coupling between gas and dust. Thus, the low value of $\alpha$ we derive under the assumption of a gas-to-dust ratio of 100 may already indicate that the gas-to-dust ratio in reality is much lower.

In our model we have $\sim 10^{-3} M_{\odot}$ of water ice in total. However, at a wavelength of $\sim 50 \mu \mathrm{m}$ the disk is not optically thin. This implies that we cannot see the entire outer disk. To estimate the amount of mass needed to account for the observed ice features we compute the total dust mass above the $\tau_{50 \mu \mathrm{m}}=1$ surface. In our model this is approximately $3 \times 10^{-5} M_{\odot}$. If we also take into account that at this wavelength only grains smaller than $20 \mu \mathrm{m}$ in size (i.e., $2 \pi a / \lambda<1$ ) and warmer than $30 \mathrm{~K}$ contribute significantly to the formation of the ice features, we obtain a contributing mass at this wavelength of $3 \times 10^{-6} M_{\odot}$. Roughly half of this mass must be in crystalline water ice, which is about 0.5 Earth masses of ice. This is the hard lower limit on the mass in crystalline water ice needed to account for the observed infrared features. From our model we also find that, because of settling, the grain size distribution above the $\tau_{50 \mu \mathrm{m}}=1$ surface is heavily depleted in grains with sizes $a>5 \mu \mathrm{m}$.

\subsection{Composition of the ice and dust}

From the ice measurements of Smith et al. (1994) along with our dust opacity computations, we can derive the peak position of the $43 \mu \mathrm{m}$ feature as a function of temperature. A very simple linear relation between the measured peak position and measurement temperature is found,

$\lambda_{\text {peak }}^{43 \mu \mathrm{m}}=\left(42.5+\frac{T}{65 \mathrm{~K}}\right) \mu \mathrm{m}$.

This relation is dependent on the dust grain model employed to convert the refractive indices into emissivities. For the $62 \mu \mathrm{m}$ feature we find a similar relation, although because of the large width of the feature this is not as accurate,

$\lambda_{\text {peak }}^{62 \mu \mathrm{m}}=\left(60.7+\frac{T}{53 \mathrm{~K}}\right) \mu \mathrm{m}$.

From the ISO spectrum we find a peak position of the ice around HD 142527 of $\lambda_{\text {peak }}^{43 \mu \mathrm{m}}=43.2 \pm 0.2 \mu \mathrm{m}$. Using Eq. (7) this corresponds to $T=45.5 \pm 13 \mathrm{~K}$. This is colder than the crystallization temperature of ice, and is also colder than the temperature of the material in the gap wall, which is around $70-80 \mathrm{~K}$. We find $\lambda_{\text {peak }}^{62 \mu \mathrm{m}}=62 \pm 0.7 \mu \mathrm{m}$ from the PACS spectrum, which corresponds to $T=69 \pm 37 \mathrm{~K}$ using Eq. (8). Because of the broadness of this feature, its spectral location is much more difficult to determine, but it is within error consistent with the low temperature derived from the $43 \mu \mathrm{m}$ feature.

In previous reductions of the ISO spectrum of HD 142527 one could identify a broad feature around $100 \mu \mathrm{m}$. The proposed carriers of this feature were carbonates or hydrous silicates (Malfait et al. 1999; Chiavassa et al. 2005), however, the feature is not detected in the PACS spectrum, which is consistent with a careful reanalysis of the ISO LWS spectrum. Therefore, we conclude there is no evidence for these species in this source.

To put constraints on the abundance of carbonates and hydrous silicates in the outer disk of HD 142527, we follow the linear least-squares fitting procedure described in Sect. 3.6. Indeed, none of the carbonates or hydrous silicates are significantly detected. Adding these components to the fitting procedure actually increases the reduced $\chi^{2}$. See Fig. 4 for the best fit we find. This fit follows the PACS spectrum more closely than the ISO spectrum because of the superior quality of the spectrum (and thus lower error bars). For water ice, we significantly detect 
Table 3. Formal upper limits of the components not significantly detected in the fitting procedure.

\begin{tabular}{lc}
\hline \hline Name & Upper limit \\
\hline Water ice - direct deposit & $40 \%$ of $M_{\text {ice }}$ \\
Water ice - warmup & $40 \%$ of $M_{\text {ice }}$ \\
\hline Montmorillonite & $47 \%$ of $M_{\text {dust }}$ \\
Serpentine & $29 \%$ of $M_{\text {dust }}$ \\
Chlorite & $8 \%$ of $M_{\text {dust }}$ \\
Dolomite & $3 \%$ of $M_{\text {dust }}$ \\
Calcite & $2 \%$ of $M_{\text {dust }}$ \\
\hline
\end{tabular}

Notes. The upper limits are derived for inclusion of single components to the best fit. For example, either the direct deposit or the warmup ice components can be added with an abundance of $40 \%$ compared to the cooldown ice component. The dust mass $M_{\text {dust }}$ includes the mass in the crystalline ice component.

only the cooldown series in the spectrum. Thus, the ice in the outer disk is compatible with being fully crystalline. In Table 3 we summarize the upper limits found for the other components. The high values for montmorillonite and serpentine are caused by their very weak spectral signature, which makes them act as a continuum component in this wavelength range. This means that although there is no indication for the presence of these materials in the spectrum, we cannot rule out their presence. For montmorillonite it was shown that at low temperatures (around $10 \mathrm{~K}$ ) the features are significantly stronger and sharper, which would reduce the upper limit on the montmorillonite (Mutschke et al. 2008).

\subsection{Scattered light images}

Although we did not use imaging data in our fitting procedure, another constraint on the scattered light of the HD 142527 outer disk comes from polarimetric imaging using NACO at the VLT (Avenhaus et al. 2014). We produce a synthetic image, which we smooth using a point spread function typical for the VLT with active adaptive optics. The resulting degree of polarization image is shown in Fig. 6. We find that in our model image the asymmetry from the observations and the overall degree of polarization are reproduced very well (compare to Avenhaus et al. 2014). The inner disk is not visible in the polarimetric image since it is very small and its polarization signal integrated over the spatial extend of the point spread function is negligible.

The $3 \mu \mathrm{m}$ resonance of water ice, which is abundantly observed in extinction in molecular clouds, causes a dip in the scattering efficiency of moderately large grains. Honda et al. (2009) took images of the outer disk in different filters in the nearinfrared. Using filters in and outside of the $3 \mu \mathrm{m}$ ice band they found a depression around $3 \mu \mathrm{m}$, which is typical for scattering off ice covered grains. Thus, there must be icy grains on the surface of the outer disk. To compare with these observations, we created a spectrum of our model in which we remove emission from the inner 0.5 " and, therefore, we only simulate and detect flux from the outer disk. We indeed detect the scattered light ice feature very clearly as is shown in Fig. 5.

\section{Discussion}

\subsection{Abundance of water ice}

We presented the detection of water ice with ISO and Herschel in the protoplanetary disk surrounding HD 142527. The increase

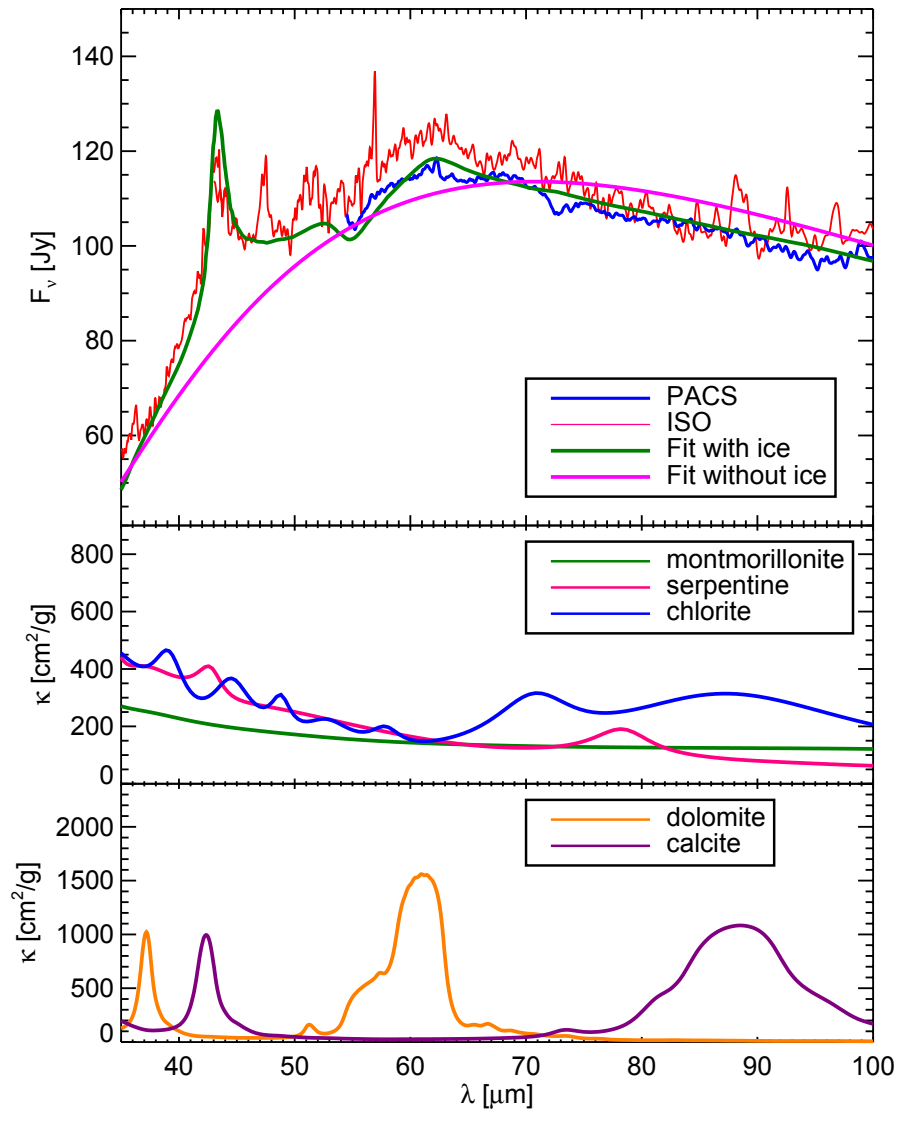

Fig. 4. Zoom in of the 35 to $100 \mu \mathrm{m}$ part of the spectrum together with the mass absorption coefficient of a selection of proposed materials (carbonates and hydrous silicates). In the upper panel we also show fits with and without crystalline water ice to highlight the significance of this ice component.

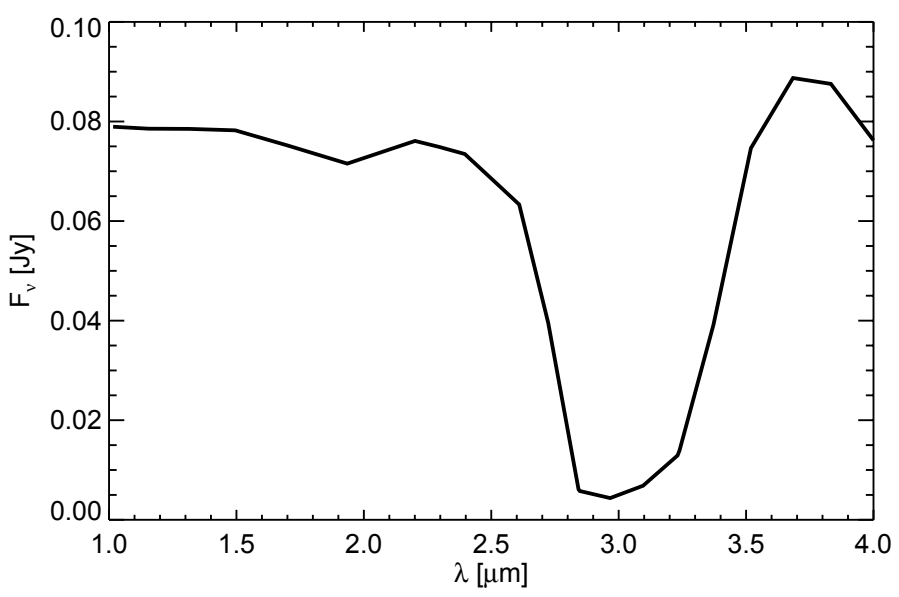

Fig. 5. Model spectrum of the outer disk. The $3 \mu \mathrm{m}$ ice feature, as detected by Honda et al. (2009), is clearly seen.

of the mass in solid material in the region where there is ice compared to where there is no ice has been estimated to range from a factor of 1.6 (see, e.g., Min et al. 2011) to a factor of 4.2 (see, e.g., Thommes \& Duncan 2006). Here we measure the strength of this jump directly from the ice to silicate ratio in the water ice emitting layer (i.e., indicated by the red region in Fig. 7, explained in the next section in more detail), which we determine to be $1.6_{-0.6}^{+0.9}$ (see Table 2). This value is surprisingly close to the solar system mixture value that is derived by Min et al. (2011) 
Table 4. Elemental composition of the mixture normalized to Si.

\begin{tabular}{lcccccc}
\hline \hline Name & $\mathrm{Si}$ & $\mathrm{Mg}$ & $\mathrm{Fe}$ & $\mathrm{S}$ & $\mathrm{C}$ & $\mathrm{O}$ \\
\hline Silicates & 1.0 & 0.65 & 0.35 & - & - & 3.0 \\
Carbon & - & - & - & - & $1.9^{ \pm 0.9}$ & - \\
Water ice & - & - & - & - & - & $9.9_{-3 .}^{+5.6}$ \\
\hline Total & 1.0 & 0.65 & 0.35 & 0.00 & $1.9^{ \pm 0.9}$ & $12.9^{+5.6}$ \\
Solar $^{a}$ & 1.0 & 1.23 & 0.98 & 0.41 & 8.3 & $15.1^{-3.7}$ \\
\hline Remaining & 0.0 & 0.58 & 0.63 & 0.41 & $6.4^{ \pm 0.9}$ & $2.2_{-5.6}^{+3.7}$ \\
\hline
\end{tabular}

Notes. ${ }^{(a)}$ Solar abundances taken from (Asplund et al. 2009) .

based on solar abundance arguments. From the abundances of all dust and ice species in our model fit, we can compute the elemental composition and compare this to the solar composition. In Table 4 we compute the fraction of all elements locked up in the different components of the model fit. Here we assume that all $\mathrm{Si}$ is locked in the silicates. Compared with solar abundances from Asplund et al. (2009), all the oxygen atoms are used up by the solid components. The slight shortage of oxygen atoms in our model falls easily within the error margins. The number of $\mathrm{Fe}$ and $\mathrm{S}$ atoms remaining is approximately equal, which is consistent with the findings in our solar system that sulfur is usually locked up in solid FeS (a dust component that is not considered in our analysis). We are, however, left with a significant $\mathrm{Mg}$ abundance, which indicates that the silicate composition as used in our model is likely not complete and should be significantly more $\mathrm{Mg}$ rich. The compositional analysis of the silicates by van Boekel et al. (2005), from which we took our silicate mixture, does not include a detailed analysis of the amorphous component in terms of $\mathrm{Fe} / \mathrm{Mg}$ ratio. An attempt to get a handle on the $\mathrm{Mg}$ content of the amorphous silicates in disks was performed by Juhász et al. (2010), who find that Mg rich silicates provide a better match to the Spitzer spectra. The conclusion is that the mixture we find is consistent with elemental abundance constraints and that roughly $77_{-7}^{+10} \%$ of the available oxygen atoms are locked up in water ice.

Here we find an ice/silicate ratio that accounts for all the oxygen and, thus, is the maximum ice reservoir one can imagine. In previous studies, the abundance of ice has been estimated to be significantly below that, leading to speculations of a vertically varying ice abundance due to enhanced grain growth from icy grains and subsequent settling of these grains below the visible surface of the disk. For example, our conclusion differs from the estimates of the ice abundance by McClure et al. (2015), who estimated that only half of the predicted ice was accounted for in the spectra of T Tauri disks. A careful reanalysis of the low $\mathrm{S} / \mathrm{N}$ features used in that study seems justified in the light of our ice abundance estimates to examine the significance of the difference. Another possibility is that this difference is somehow related to the complex, dynamical geometry of the system, which should be a topic of future study.

\subsection{Origin of the crystallinity of the ice}

We have shown that the surface layer of the outer disk of HD 142527 contains a large abundance of crystalline water ice. Crystalline water ice forms in the disk at temperatures above $\sim 90 \mathrm{~K}$, which is warmer than the temperature where we detect it, which is around $45 \mathrm{~K}$. We derive an upper limit of $40 \%$ of the ice to be in amorphous form (see Table 3), but the modeling fit is also consistent with $100 \%$ crystalline ice.

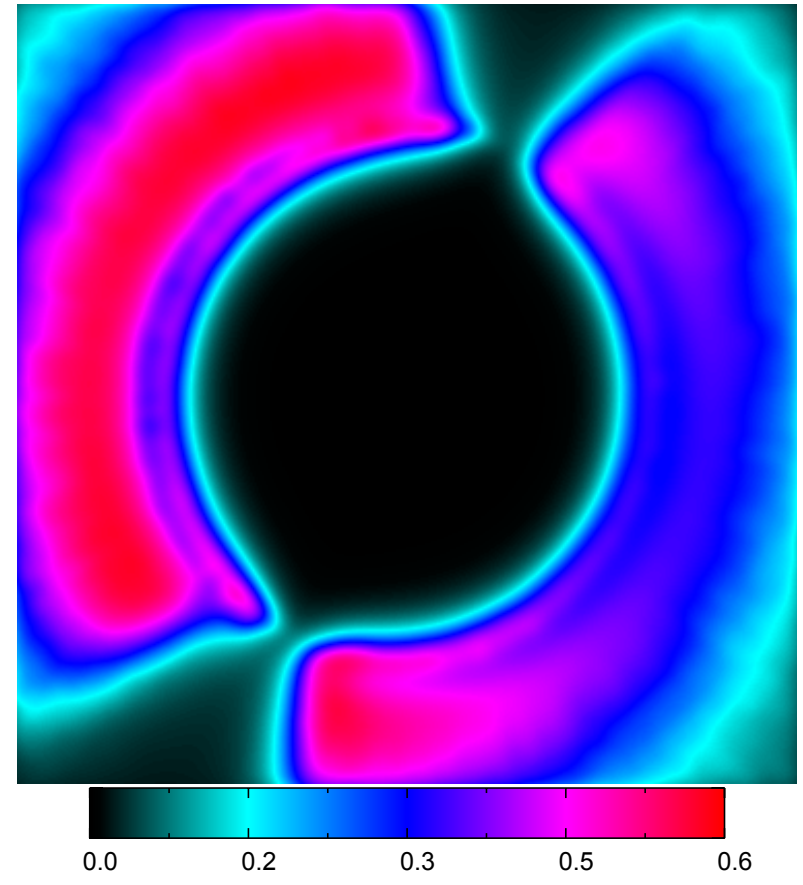

Fig. 6. Degree of linear polarization predicted by our HD 142527 model to be observed with VLT-NACO. The values compare very well with those obtained by Avenhaus et al. (2014). The field of view of the image is 2.85 arcsec.

\subsection{Crystallization and origin of ice}

The conditions in a laboratory on Earth are different from the conditions in a protoplanetary disk. In particular, the available timescales are significantly longer in a protoplanetary disk. Therefore, we have to carefully consider what happens to the ice when it stays at a certain temperature for a longer period of time. In the laboratory a crystallization temperature of $110 \mathrm{~K}$ is typically obtained for a crystallization timescale on the order of 1 day. The temperature needed for crystallization decreases if one allows more time for the transition to occur. Using the equation for the temperature dependent crystal growth rate from Jenniskens \& Blake (1996) the timescale for crystallization scales as,

$t_{\text {disk }}=t_{\text {lab }} \exp \left(-\left(E_{\mathrm{a}} / R\right)\left\{\frac{1}{T_{\text {lab }}}-\frac{1}{T_{\text {disk }}}\right\}\right)$.

Here $t_{\text {disk }}$ and $t_{\text {lab }}$ are the crystallization timescale in the disk and in the laboratory, respectively, $T_{\text {disk }}$ and $T_{\text {lab }}$ are the crystallization temperatures in the disk and the laboratory, $E_{\mathrm{a}}$ is the crystallization activation energy (taken to be $E_{\mathrm{a}}=59.2 \mathrm{~kJ} / \mathrm{mol}$ after Baragiola et al. 2009), and $R$ is the gas constant $(R=$ $\left.8.314 \times 10^{-3} \mathrm{~kJ} / \mathrm{mol} / \mathrm{K}\right)$. We have to insert the local dynamical timescale for the disk, since this is the typical timescale that a dust grain can spend at a given location. Most dynamical processes act on a timescale on the order of the orbital timescale. At the inner edge of the outer wall (a radius of $130 \mathrm{AU}$ ) this timescale is typically $1000 \mathrm{yr}$, corresponding to a crystallization temperature of $T_{\text {disk }}=91 \mathrm{~K}$ instead of the $110 \mathrm{~K}$ measured in the laboratory.

In order to be able to plot some key features of the ice and temperature structure in the disk, we remove the inner disk to obtain a circularly symmetric model of the outer disk that we can map onto a 2D figure. Removing the inner disk implies that we are basically considering here the nonshadowed parts of the outer disk alone. In the left panel of Fig. 7 we plot the region 

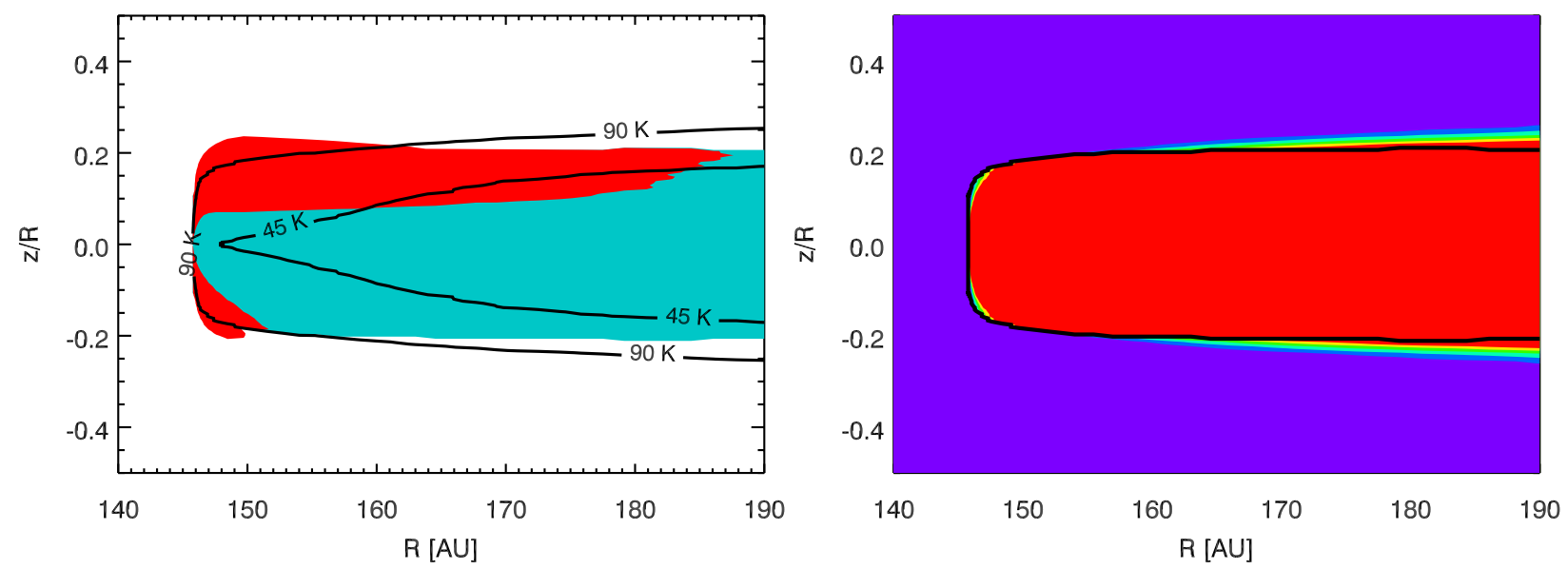

Fig. 7. Left panel: red area indicates the region in the disk where $90 \%$ of the flux at $43 \mu \mathrm{m}$ originates. The blue area is the region where ice can exist, i.e., the temperature is low enough, density high enough, and UV field weak enough. The contours show the temperature of the ice according to the feature position $(45 \mathrm{~K})$ and the crystallization temperature at one orbital timescale. Right panel: number of orbits required for the ice to crystallize. Colorscale: less than an orbit (purple), between 1 and 10 orbits (dark blue), between 10 and 100 orbits (light blue), between 100 and 1000 orbits (green), between 1000 and 10000 orbits (yellow), and above 10000 orbits (red). The thick black line indicates the region where ice can exist.

in the disk where the $43 \mu \mathrm{m}$ flux originates. The red region indicates where $90 \%$ of the flux at $43 \mu \mathrm{m}$ comes from. The blue region indicates the region where in the outer disk ice can exist according to Eq. (5). We also draw contours for $45 \mathrm{~K}$ (the temperature of the ice according to the peak position) and $90 \mathrm{~K}$ (the temperature where ice can crystallize in one orbital timescale). In the right panel we plot the number of orbits required for ice to crystallize at that location in the disk according to Eq. (9) (colorscale as indicated in the caption). In that panel, the thick black line indicates the location where ice can exist. As can be seen from these figures, by far most of the ice is in a region where it is expected to be amorphous. Also, most of the flux at $43 \mu \mathrm{m}$ originates in this region. We now consider a few options for generating the amount of crystalline ice seen in the outer disk.

Condensation in the gap: at a distance of $\sim 100 \mathrm{AU}$ from the star, the temperature of the dust grains in the optically thin limit reaches $100 \mathrm{~K}$, which is sufficient to make crystalline water ice. However, this is right inside the gap. The computations performed with ProDiMo confirmed that the vapor pressure of $\mathrm{H}_{2} \mathrm{O}$ here is too low to form water ice (see also Fig. 7).

In situ formation in the outer disk: in the outer disk, ice condensation can take place. As mentioned before, at the equilibrium temperature at that location the ice forms amorphously. In Fig. 7 it can be seen that there are small regions where the ice could crystallize in 10 to 100 orbits. These are regions high up in the disk, however, where the densities are low, so there is not much ice there. Crystallizing the entire region responsible for the $43 \mu \mathrm{m}$ feature requires substantial mixing to process the entire material in a column through the small crystallization region. This basically implies that we have to divide the timescale required for crystallization by the fraction of the ice that actually is at high enough temperatures to compute the timescale for full crystallization. This results in a timescale that is significantly longer than the lifetime of the disk. The crystalline ice we detect therefore requires (transient) heating above $100 \mathrm{~K}$, where the ice crystallizes almost instantaneously. These events have to heat the material somewhere between 100 and $160 \mathrm{~K}$ such that the ice crystallizes without evaporating. Alternatively, the heating can be to temperatures above $160 \mathrm{~K}$, where the ice evaporates and the cooling has to be sufficiently slow to allow the ice to reform crystalline. If the source of the heating is a transient accretion burst, we can compute the temperature structure using the method of Min et al. (2011). We find that an accretion burst of $10^{-4} M_{\odot} / \mathrm{yr}$, which is very high, provides enough energy (both locally produced and through irradiation from the inner accretion shock) to crystallize the ice.

Heating by a companion inside the gap: one of the possible causes of the gap is the presence of a companion close to the inner edge of the outer disk. The luminosity of this companion can heat up the dust grains in the wall of the outer disk when it passes. If we assume that the companion is one Hill radius away from the outer edge of the gap, which is at least around $10 \mathrm{AU}$ at this distance from the star, the required luminosity of the companion to heat the grains from 80 to $100 \mathrm{~K}$ is around $8 \times 10^{-3} L_{\star}$. This is almost two orders of magnitude above the detection limits obtained by Rameau et al. (2012), which rules out this scenario.

Transportation from the inner disk: in the midplane of the inner disk, the temperature and densities are right for creating crystalline water ice. At the outer edge of the inner disk, around 30$40 \mathrm{AU}$, the temperature at the surface of the disk is low enough for the ice to survive for a moderate amount of time. One could imagine the crystalline ice being created there and transported through the gap by the radiation pressure from the star (similar to the mechanism proposed by Vinković 2009). Normally in a protoplanetary disk, radiation pressure cannot move the grains very far because they are stopped by the gas. In this case, however, transportation through the low density region, the gap, seems possible depending on the currently unknown gas density inside the gap.

Creation in the gap by collisions: inside a larger body, the water ice can be preserved and protected against evaporation, photodestruction, and amorphization. Indeed, the ice in Kuiper Belt objects can be crystalline (Jewitt \& Luu 2004), even at the surface. The gap in the disk is very large, and deep imaging has so far only revealed upper limits to possible planetary companions inside the gap (Rameau et al. 2012). If this very large gap is created by multiple planets, it can very well be that it also houses a collection of large planetesimals. When these bodies collide, they can free their crystalline interior in the form of small dust grains, which are pushed toward the outer disk by radiation pressure or a stellar wind. This mechanism was also proposed to explain the presence of crystalline forsterite particles at the inner edge of the outer disk in the HD 100546 system 
(Bouwman et al. 2003; Mulders et al. 2011) and the crystalline water ice in T Tauri disks (McClure et al. 2015). The 0.5 Earth masses of water ice correspond to roughly $5 \times 10^{7} 1-\mathrm{km}$-sized planetesimals.

No matter what the formation mechanism is, the detection of crystalline water ice is consistent with observations on solar system satellites, where most are detected to have crystalline ice at least at the surface (Mastrapa et al. 2013); this is the case for satellites that seem too cold to have crystalline ice as well.

\section{Conclusions}

We report on the far-infrared spectrum of the Herbig star HD 142527. The Herschel PACS spectrum of this source does not confirm the previous claim of a $105 \mu \mathrm{m}$ feature in the ISO LWS spectrum. We identify calibration issues with the ISO LWS spectrum as the source of the confusion. The $62 \mu \mathrm{m}$ water ice feature is clearly detected in the PACS spectrum, as is the $43 \mu \mathrm{m}$ water ice feature in the ISO SWS spectrum.

The full SED of the source is modeled in detail with a special focus on the detection of crystalline water ice. Assuming a homogeneous composition of the outer disk, and ice condensation where possible, we infer an ice/silicate ratio of $1.6_{-0.6}^{+0.9}$. This implies that roughly $80 \%$ of the available oxygen atoms are locked up in water ice. We compute the elemental abundances of the derived dust/ice mixture and conclude that it is consistent with solar abundance constraints. Furthermore, from our modeling we find that the scale height of the disk in the inner regions is significantly above hydrostatic equilibrium, while in the outer parts of the disk it is close to hydrostatic equilibrium. This is including detailed settling with a very low value of the turbulent mixing strength, $\alpha$.

We identify crystalline water ice in a location of the disk where equilibrium temperatures are too low to form crystalline ice in situ. In addition, the spectral position of the $43 \mu \mathrm{m}$ ice feature is indicative of ice at a temperature of $45 \mathrm{~K}$. At these temperatures amorphous ice is expected to form. We discuss possible scenarios for forming or transporting the crystalline ice there. We conclude that a plausible scenario is formation inside the large $146 \mathrm{AU}$ gap through collisions of icy planetesimals. Another possibility is crystallization through extreme accretion events liberating enough energy to heat up and crystallize the ice in the outer regions.

Our findings of a large region in the disk where the solid mass is dominated by water ice is promising for planet formation models that require water ice as a mechanism for increasing the dust mass and sticking probability. It also provides a huge reservoir of volatile elements that may contribute at a later stage to the enrichment of dry planets closer to the star.

Acknowledgements. We would like to thank Inga Kamp for valuable discussions on the influence of UV radiation. Support for this work, part of the Herschel Open Time Key Project Program, was provided by NASA through an award issued by the Jet Propulsion Laboratory, California Institute of Technology. M.M. acknowledges funding from the EU FP7- 2011 under Grant Agreement No. 284405. OSIA is a joint development of the SWS consortium. Contributing institutes are SRON, MPE, KUL, and the ESA Astrophysics Division. The ISO Spectral Analysis Package (ISAP) is a joint development by the LWS and SWS Instrument Teams and Data Centers. Contributing institutes are CESR, IAS, IPAC, MPE, RAL, and SRON.

\section{References}

Asplund, M., Grevesse, N., Sauval, A. J., \& Scott, P. 2009, ARA\&A, 47, 481 Avenhaus, H., Quanz, S. P., Schmid, H. M., et al. 2014, ApJ, 781, 87 Bans, A., \& Königl, A. 2012, ApJ, 758, 100
Baragiola, R. A., Burke, D. J., \& Fama, M. A. 2009, AGU Fall Meeting Abstracts Biller, B., Lacour, S., Juhász, A., et al. 2012, ApJ, 753, L38

Birnstiel, T., Dullemond, C. P., \& Pinilla, P. 2013, A\&A, 550, L8

Bouwman, J., de Koter, A., Dominik, C., \& Waters, L. B. F. M. 2003, A\&A, 401, 577

Canovas, H., Ménard, F., Hales, A., et al. 2013, A\&A, 556, A123

Casassus, S., van der Plas, G., M, S. P., et al. 2013, Nature, 493, 191

Charbonneau, P. 1995, ApJS, 101, 309

Chiang, E. I., Joung, M. K., Creech-Eakman, M. J., et al. 2001, ApJ, 547, 1077

Chiavassa, A., Ceccarelli, C., Tielens, A. G. G. M., Caux, E., \& Maret, S. 2005, A\&A, 432, 547

Chihara, H., Koike, C., \& Tsuchiyama, A. 2001, PASJ, 53, 243

Christiaens, V., Casassus, S., Perez, S., van der Plas, G., \& Ménard, F. 2014, ApJ, $785, \mathrm{~L} 12$

Clegg, P. E., Ade, P. A. R., Armand, C., et al. 1996, A\&A, 315, L38

Close, L. M., Follette, K. B., Males, J. R., et al. 2014, ApJ, 781, L30

Creech-Eakman, M. J., Chiang, E. I., Joung, R. M. K., Blake, G. A., \& van Dishoeck, E. F. 2002, A\&A, 385, 546

de Graauw, T., Haser, L. N., Beintema, D. A., et al. 1996, A\&A, 315, L49

Dorschner, J., Begemann, B., Henning, T., Jäger, C., \& Mutschke, H. 1995, A\&A, 300, 503

Dubrulle, B., Morfill, G., \& Sterzik, M. 1995, Icarus, 114, 237

Fabian, D., Henning, T., Jäger, C., et al. 2001, A\&A, 378, 228

Ferrarotti, A., Gail, H.-P., Degiorgi, L., \& Ott, H. R. 2000, A\&A, 357, L13

Green, J. D., Yang, Y.-L., Evans, II, N. J., et al. 2016, AJ, 151, 75

Habing, H. J. 1968, Bull. Astron. Inst. Netherlands, 19, 421

Hartmann, L., Calvet, N., Gullbring, E., \& D'Alessio, P. 1998, ApJ, 495, 385

Honda, M., Inoue, A. K., Fukagawa, M., et al. 2009, ApJ, 690, L110

Hughes, A. M., Wilner, D. J., Qi, C., \& Hogerheijde, M. R. 2008, ApJ, 678, 1119

Jäger, C., Molster, F. J., Dorschner, J., et al. 1998, A\&A, 339, 904

Jenniskens, P., \& Blake, D. F. 1996, ApJ, 473, 1104

Jewitt, D. C., \& Luu, J. 2004, Nature, 432, 731

Juhász, A., Bouwman, J., Henning, T., et al. 2010, ApJ, 721, 431

Kama, M., Min, M., \& Dominik, C. 2009, A\&A, 506, 1199

Kessler, M. F., Steinz, J. A., Anderegg, M. E., et al. 1996, A\&A, 315, L27

Klahr, H. H., \& Henning, T. 1997, Icarus, 128, 213

Koike, C., \& Shibai, H. 1990, MNRAS, 246, 332

Lacour, S., Biller, B., Cheetham, A., et al. 2016, A\&A, 590, A90

Maaskant, K. M., Honda, M., Waters, L. B. F. M., et al. 2013, A\&A, 555, A64

Malfait, K., Waelkens, C., Bouwman, J., de Koter, A., \& Waters, L. B. F. M. 1999, A\&A, 345, 181

Marino, S., Perez, S., \& Casassus, S. 2015, ApJ, 798, L44

Mastrapa, R. M. E., Grundy, W. M., \& Gudipati, M. S. 2013, in The Science of Solar System Ices, eds. M. S. Gudipati, \& J. Castillo-Rogez, Astrophys. Space Science Lib., 356, 371

McClure, M. K., Manoj, P., Calvet, N., et al. 2012, ApJ, 759, L10

McClure, M. K., Espaillat, C., Calvet, N., et al. 2015, ApJ, 799, 162

Min, M., Hovenier, J. W., \& de Koter, A. 2005, A\&A, 432, 909

Min, M., Waters, L. B. F. M., de Koter, A., et al. 2007, A\&A, 462, 667

Min, M., Dullemond, C. P., Dominik, C., de Koter, A., \& Hovenier, J. W. 2009, A\&A, 497, 155

Min, M., Dullemond, C. P., Kama, M., \& Dominik, C. 2011, Icarus, 212, 416

Mulders, G. D., Waters, L. B. F. M., Dominik, C., et al. 2011, A\&A, 531, A93

Müller, T., Balog, Z., Nielbock, M., et al. 2014, Exper. Astron., 37, 253

Mutschke, H., Zeidler, S., Posch, T., et al. 2008, A\&A, 492, 117

Ott, S. 2010, in Astronomical Data Analysis Software and Systems XIX, eds. Y. Mizumoto, K.-I. Morita, \& M. Ohishi, ASP Conf. Ser., 434, 139

Poglitsch, A., Waelkens, C., Geis, N., et al. 2010, A\&A, 518, L2

Pollack, J. B., Hollenbach, D., Beckwith, S., et al. 1994, ApJ, 421, 615

Posch, T., Baier, A., Mutschke, H., \& Henning, T. 2007, ApJ, 668, 993

Preibisch, T., Ossenkopf, V., Yorke, H. W., \& Henning, T. 1993, A\&A, 279, 577

Rameau, J., Chauvin, G., Lagrange, A.-M., et al. 2012, A\&A, 546, A24

Servoin, J. L. \& Piriou, B. 1973, Physica Status Solidi B Basic Research, 55, 677

Smith, R. G., Robinson, G., Hyland, A. R., \& Carpenter, G. L. 1994, MNRAS, 271,481

Spitzer, W. G., \& Kleinman, D. A. 1960, Phys. Rev., 121, 1324

Thommes, E. W., \& Duncan, M. J. 2006, in Planet Formation, The accretion of giant-planet cores, eds. H. Klahr, \& W. Brandner (Cambridge University Press), 129

van Boekel, R., Min, M., Leinert, C., et al. 2004, Nature, 432, 479

van Boekel, R., Min, M., Waters, L. B. F. M., et al. 2005, A\&A, 437, 189

van der Plas, G., Casassus, S., Menard, F., et al. 2014, ApJ, 792, L25

van der Wiel, M. H. D., Naylor, D. A., Kamp, I., et al. 2014, MNRAS, 444, 3911

Verhoeff, A. P., Min, M., Pantin, E., et al. 2011, A\&A, 528, A91

Vinković, D. 2009, Nature, 459, 227

Woitke, P., Kamp, I., \& Thi, W.-F. 2009, A\&A, 501, 383

Woitke, P., Min, M., Pinte, C., et al. 2016, A\&A, 586, A103 